\title{
Physical mechanism of the linear beam-size effect at colliders
}

\author{
K. Melnikov \\ Institut für Physik,Universität Mainz* \\ and \\ G. L. Kotkin and V.G. Serbo \\ Novosibirsk State University
}

August 23, 2021

\begin{abstract}
We present qualitative but precise description of the linear beam-size effect predicted for the processes in which unstable but long-living particles collide with each other. We derive physically pronounced equation for the events rate which proves that the linear beam-size effect corresponds to the scattering of one beam of particles on the decay products of the other. We compare this linear beam-size effect with the known logarithmic beam-size effect measured in the experiments on a single bremsstrahlung at VEPP-4 and HERA.
\end{abstract}

MZ-TH/96-11

${ }^{*}$ D 55099 Germany, Mainz, Johannes Gutenberg Universität, Institut für Physik, THEP, Staudinger weg 7; e-mail: melnikov@dipmza.physik.uni-mainz.de

${ }^{\dagger}$ Novosibirsk State University, 630090, Novosibirsk, Russia; e-mail: kotkin@phys.nsu.nsk.su; serbo@math.nsk.su 


\section{Introduction}

Let us consider a process with unstable but long-living particle(s) in the initial state. Calculating corresponding cross section, one can meet a singularity in the physical region related to the appearance of real (not virtual) particles in an intermediate state. The appearance of such singularities leads to a divergent cross section. To perform calculations in this case a careful analyses of the physical set-up is needed.

Such situation takes place for a number of processes at muon colliders, for instance, $\mu^{+} \mu^{-} \rightarrow e \bar{\nu}_{e} X, \mu^{+} \mu^{-} \rightarrow e \nu_{\mu} X, \ldots$ which have been recently discussed in refs. [1], 2]. To be concrete, below we consider the process

$$
\mu^{-} \mu^{+} \rightarrow e \bar{\nu}_{e} W^{+} .
$$

One of the Feynman diagrams of this process which contains the abovementioned singularity (the so-called $t$-channel singularity in the physical region) is presented in the Fig.1. This diagram can be viewed as a sequence of two processes:

$$
\mu^{-} \rightarrow e \bar{\nu}_{e} \nu_{\mu}
$$

and

$$
\nu_{\mu} \mu^{+} \rightarrow W^{+}
$$

both of which can occur for the real muonic neutrino. Therefore, there exists a region of the final phase space corresponding to the reaction (1) where denominator of the propagator of the muonic neutrino $q^{2}$ can be equal to zero t.

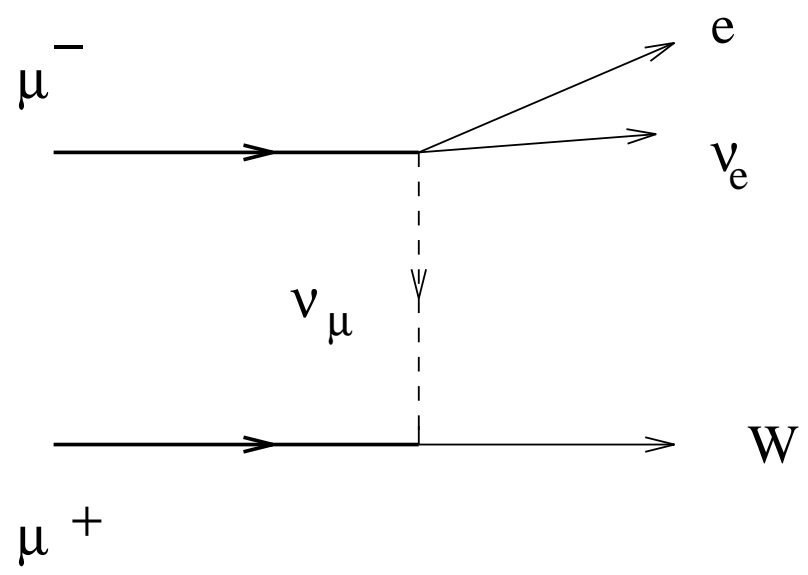

Figure 1: The Feynman diagram for the reaction $\mu^{-} \mu^{+} \rightarrow e \bar{\nu}_{e} W^{+}$which has the $t^{-}$ channel singularity and gives the leading contribution in the region of small $\left|q^{2}\right|$.

In the paper [2] it has been shown that accounting for the finite sizes of the colliding beams gives a finite cross section for the processes with the $t$-channel singularity in the physical region. This result has been obtained on the base of the results of the paper [3] where a general technique for taking into account the finite sizes of the colliding beams has been worked out (for the review see [4]). The effective cross section obtained in [2]

$$
d \sigma=\frac{d N}{L}
$$

\footnotetext{
${ }^{1}$ For the appearance of such singularity it is necessary that both of the processes (2, 3) have a possibility to occur.
} 
(where $N$ is the number of events and $L$ is the luminosity of the $\mu^{+} \mu^{-}$collisions) can be written in the form

$$
d \sigma=d \sigma_{\mathrm{st}}+d \sigma_{\mathrm{nst}} .
$$

The cross section $d \sigma_{\mathrm{st}}$ by definition corresponds to the wide region of the phase space defined by the inequality $q^{2}<-m^{2}$ where $m$ is the muon mass. This part of the cross section can be calculated by means of the standard rules of the relativistic scattering theory. For the discussed process the result of this calculation can be found in [2]. The cross section $d \sigma_{\text {nst }}$ corresponds to the narrow region of the final phase space where

$$
-m^{2}<q^{2}<t_{0}, \quad t_{0}>0
$$

which includes the point $q^{2}=0$. It turns out [2] that the main contribution to this cross section is given by the pole of the $\nu_{\mu}$ propagator and that this contribution is proportional to the transverse size of the colliding beams $a$ :

$$
d \sigma_{\mathrm{nst}} \propto a .
$$

It is the linear beam-size effect.

In the present paper we show that the non-standard piece of the cross section $d \sigma_{\text {nst }}$ is defined by the sequence of processes (2), (3) for real muonic neutrinos. Such interpretation of the results of ref. [2] leads, in particular, to a more clear understanding of its region of applicability.

In view of the realistic situation at future muon colliders, we assume that longitudinal $l$ and transverse $a$ sizes of the colliding beams satisfy the inequalities:

$$
\begin{gathered}
l \ll \gamma c \tau, \quad \gamma=\frac{E}{m c^{2}}, \\
a \ll c \tau
\end{gathered}
$$

where $\tau$ is the muon life time in its rest frame, $c \tau=660 \mathrm{~m}$. Due to the condition (8) the number of muons can be thought to be a constant during the time which is required for the colliding beams to cross each other. Note, that conditions (8)-(9) are perfectly fulfilled for the projects of muon colliders which are discussed in the literature [5]. Using the beam parameters from ref. [5] and the total energy $2 E=100 \mathrm{GeV}$ one gets:

$$
\frac{a}{c \tau} \sim \frac{l}{\gamma c \tau} \sim 10^{-8}
$$

Subsequent part of the paper is organized as follows: in the next section we give a simple qualitative description of the linear beam-size effect; section 3 is devoted to the direct calculation of the number of events corresponding to the $\nu_{\mu} \mu^{+}$collisions; in section 4 we transform the result derived in ref. [2] for $\sigma_{\text {nst }}$ to the rest frame of the $\mu^{-}$beam and compare it with the results of section 3 ; in section 5 we discuss a case when conditions (8)-(9) are not satisfied; our conclusions are presented in section 6 .

\section{Qualitative description of the linear beam-size ef- fect}

We consider a collision of a single muon $\mu^{-}$with the beam of muons of the positive charge $\mu^{+}$. In this analysis it is convenient to use the rest frame of the $\mu^{-}$. Being unstable, 
this muon is surrounded by a "cloud" of $\nu_{\mu}$ 's which appear in the $\mu^{-}$decay (certainly, there are also $\bar{\nu}_{e}$ and $e$ "clouds", but they are not interesting for us at the moment). The density of neutrinos in this cloud decreases as $1 / r^{2}$ with the growth of the distance $r$ from the $\mu^{-}$, its time dependence corresponds to the exponential decay law and the angular distribution is isotropic. Therefore, one finds

$$
n_{\nu}(\mathbf{r}, t)=\frac{\theta(t-r / c)}{4 \pi c \tau r^{2}} \exp \left(-\frac{t-r / c}{\tau}\right) .
$$

This density is normalized by the condition

$$
\int_{0}^{\infty} c n_{\nu}(\mathbf{r}, t) 4 \pi r^{2} d t=1
$$

which means that the total number of $\nu_{\mu}$ 's which cross a sphere of radius $r$ is equal to unity.

We shall see below that the main contribution to the $\sigma_{\text {nst }}$ comes from the distance $r \sim a$. The typical time of collision is therefore $\Delta t \sim a / c$. If the collision occurs at the time $t$ which satisfies the inequality $\Delta t \ll t \ll \tau$, the neutrino density can be taken to be time independent in the collision region (these assumptions are justified by the obtained result):

$$
n_{\nu}(\mathbf{r}, t)=\frac{1}{4 \pi c \tau r^{2}}
$$

The distribution of the neutrinos over impact parameters $\varrho$ of the $\mu^{+} \mu^{-}$collisions @ is given by

$$
d N_{\nu}=\frac{d^{2} \varrho}{4 \pi c \tau} \int_{-\infty}^{+\infty} \frac{d z}{z^{2}+\varrho^{2}}=\frac{1}{4 c \tau} \frac{d^{2} \varrho}{\varrho}, \quad \mathbf{r}=(\varrho, z)
$$

We note that the main contribution to this integral is given by the longitudinal distances of the order of

$$
|z| \lesssim \varrho
$$

The number of muonic neutrinos which collide with the $\mu^{+}$beam of the radius $a$ is therefore:

$$
N_{\nu} \sim \int_{0}^{a} \frac{d^{2} \varrho}{4 c \tau \varrho}=\frac{\pi}{2} \frac{a}{c \tau} .
$$

The non-standard piece of the cross section of the process (1) is proportional to the number of neutrinos (14) and can be estimated as:

$$
\sigma_{\mathrm{nst}} \sim \frac{a}{c \tau}\left\langle\sigma_{\nu \mu \rightarrow W}\right\rangle
$$

where $\left\langle\sigma_{\nu \mu \rightarrow W}\right\rangle$ corresponds to the cross section of the process (3) averaged over effective $\nu_{\mu}$ spectrum 阝 $^{\text {. }}$.

The estimate (15) corresponds to the $\sigma_{\text {nst }}$ obtained in [2]. The exact calculation is presented in the next section.

\footnotetext{
${ }^{2}$ The $z$-axis is antiparallel to the momentum $\mathbf{p}_{2}$ of the $\mu^{+}$.

${ }^{3}$ The exact form of this spectrum can be found in ref. [2].
} 


\section{Direct calculation of the number of events in $\nu_{\mu} \mu^{+}$ collisions}

Having performed the estimate, we proceed further with the exact calculation. We perform it in the rest frame of the $\mu^{-}$beam in which neutrino density $n_{\nu}$ has the simple form (12). For simplicity, we neglect energy and angular spread of the particles in the muon beams.

The number of events for the $\nu_{\mu} \mu^{+} \rightarrow W^{+}$process can be written in the form

$$
d N_{\nu \mu \rightarrow W}=d L_{\nu \mu}(\omega) \sigma_{\nu \mu \rightarrow W}\left(s_{\nu \mu}\right) .
$$

Here $d L_{\nu \mu}(\omega)$ is the spectral luminosity of $\nu_{\mu} \mu^{+}$collisions. The cross section $\sigma_{\nu \mu \rightarrow W}$ depends on the quantity

$$
s_{\nu \mu}=\left(q+p_{2}\right)^{2}=2 \omega E_{2}(1+\cos \theta)+m^{2}
$$

where $\omega$ and $\theta, \varphi$ are the energy and the escape angles of $\nu_{\mu}$, and $E_{2}$ is the energy of $\mu^{+}$.

For unpolarized muons the energy distribution of the $\nu_{\mu}$ does not depend on the neutrino escape angles and has the well-known form [6]

$$
w(\omega) d \omega=16\left(\frac{\omega}{m}\right)^{2}\left(3-\frac{4 \omega}{m}\right) \frac{d \omega}{m} .
$$

Let $n_{1}\left(\mathbf{r}_{1}\right)$ and $n_{2}\left(\mathbf{r}_{2}, t\right)$ be the particle densities of $\mu^{-}$and $\mu^{+}$beams respectively. In accordance with eq. (12) the density of the neutrinos in the point $\mathbf{r}_{2}$ is defined by the muons $\mu^{-}$decaying at the point $\mathbf{r}_{1}$ by the following equation:

$$
d n_{\nu}\left(\mathbf{r}_{2}\right)=\frac{n_{1}\left(\mathbf{r}_{1}\right) d^{3} r_{1}}{4 \pi c \tau\left(\mathbf{r}_{2}-\mathbf{r}_{1}\right)^{2}}
$$

Therefore, the spectral luminosity of $\nu_{\mu} \mu^{+}$collisions is equal to (see, for example, [7] $\S 12$ )

$$
d L_{\nu \mu}(\omega)=w(\omega) d \omega v d n_{\nu}\left(\mathbf{r}_{2}\right) n_{2}\left(\mathbf{r}_{2}, t\right) d^{3} r_{2} d t
$$

where

$$
v=\frac{q p_{2}}{\omega E_{2}}=1+\cos \theta
$$

Note that the vector $\mathbf{r}_{2}-\mathbf{r}_{1}$ is parallel to the neutrino momentum $\mathbf{q}$ :

$$
\left(\mathbf{r}_{2}-\mathbf{r}_{1}\right) \| \mathbf{q} \text {. }
$$

In the next section we shall show that the result for $d N_{\text {nst }}$ obtained in [2] completely coincides with eqs. (16)-(20).

\section{The number of events $d N_{\text {nst }}$ in the rest frame of the $\mu^{-}$beam}

In this section we follow the line of reasoning of ref. [2]. The only difference is that we switch to the rest frame of the $\mu^{-}$beam. It turns out that this reference frame is quite helpful for the physical interpretation of the results obtained in ref. [2].

\footnotetext{
${ }^{4}$ Below we use a system of units where $\hbar=1$ and $c=1$.
} 
We use the following notations (see Fig. 1): $s=\left(p_{1}+p_{2}\right)^{2}=4 E^{2}$ is the square of the total energy in the center of mass frame, $\Gamma=1 / \tau$ is the muon width, $p_{1}^{2}=p_{2}^{2}=m^{2}, p_{3}+p_{4}$ is the 4 -momentum of the final $e^{-} \bar{\nu}_{e}$ system, $y=\left(p_{3}+p_{4}\right)^{2} / m^{2}, q=p_{1}-p_{3}-p_{4}=(\omega, \mathbf{q})$ is the momentum transfer in the $t$-channel and $x=q p_{2} / p_{1} p_{2}$. The square of the momentum transfer in the $t$-channel is equal to

$$
q^{2}=-\frac{\mathbf{q}_{\perp}^{2}}{1-x}+t_{0}
$$

where $\mathbf{q}_{\perp}$ is the component of the momentum $\mathbf{q}$ which is transverse to the momenta of the initial muons and $t_{0}$ is the maximal value of $q^{2}$

$$
\max \left\{q^{2}\right\}=t_{0}=\frac{x(1-x-y)}{1-x} m^{2} .
$$

Note, that

$$
t_{0}>0 \text { for } y<1-x .
$$

As is explained in the Introduction, usual approximations in the scattering theory (based on the collisions of the plane waves) result in the divergent cross section in this case. In contrast to this approximation, we use the formalism of the colliding wave packets (beams of particles) of macroscopic, but finite sizes which has been developed in ref. [3]. In the overwhelming majority of experiments both descriptions provide the same result. However, in a number of cases the usual approximation is insufficient and consideration of the colliding wave packets is mandatory - see description of the experiments on a single bremsstrahlung at VEPP-4 [8] and at HERA [9] and the review [4]. The same can be stated about discussed processes with the singularities caused by real particles in the intermediate states ( $t$-channel singularities in the physical region). The breakdown of the standard calculations clearly indicates inapplicability of the standard approximations in the scattering theory for these cases.

Let us remind that in the standard approach the number of events $N$ is the product of the cross section $\sigma$ and the luminosity $L$ :

$$
d N=d \sigma L, \quad d \sigma \propto|M|^{2}, \quad L=v_{12} \int n_{1}(\mathbf{r}, t) n_{2}(\mathbf{r}, t) d^{3} r d t
$$

where $v_{12}=\left|\mathbf{v}_{1}-\mathbf{v}_{2}\right|=2$ for the head-on collision of the ultra-relativistic beams. The quantities $n_{i}(\mathbf{r}, t)$ are the particle densities of the beams.

The transformation from the plane waves to the wave packets results in the following changes. The squared matrix element $|M|^{2}$ with the initial state in the form of the plane waves with the momenta $\mathbf{p}_{\mathbf{1}}$ and $\mathbf{p}_{\mathbf{2}}$ transforms to the product of the matrix elements $M_{f i}$ and $M_{f i^{\prime}}^{*}$ with different initial states:

$$
d \sigma \propto|M|^{2} \rightarrow d \sigma(\kappa) \propto M_{f i} M_{f i^{\prime}}^{*} .
$$

Here the initial state $|i\rangle$ is the direct product of the plane waves with the momenta $\mathbf{k}_{1}=\mathbf{p}_{1}+\frac{1}{2} \boldsymbol{\kappa}$ and $\mathbf{k}_{2}=\mathbf{p}_{2}-\frac{1}{2} \boldsymbol{\kappa}$, while the initial state $\left|i^{\prime}\right\rangle$ is the direct product of the plane waves with the momenta $\mathbf{k}_{1}^{\prime}=\mathbf{p}_{1}-\frac{1}{2} \boldsymbol{\kappa}$ and $\mathbf{k}_{2}^{\prime}=\mathbf{p}_{2}+\frac{1}{2} \boldsymbol{\kappa}$. Instead of the luminosity $L$ the number of events starts to depend on the quantity

$$
L(\mathbf{r})=v_{12} \int n_{1}\left(\mathbf{r}_{2}-\mathbf{r}, t\right) n_{2}\left(\mathbf{r}_{2}, t\right) d^{3} r_{2} d t
$$


through the following formula

$$
d N=\int \frac{d^{3} \kappa d^{3} r}{(2 \pi)^{3}} \mathrm{e}^{i \boldsymbol{\kappa} \mathbf{r}} d \sigma(\boldsymbol{\kappa}) L(\mathbf{r})
$$

Note that in this formula the densities $n_{1}$ and $n_{2}$ are taken in different points - it corresponds to the nonlocal interaction of colliding muons (contrary, in eq. (25) these densities are taken in one and the same point).

The characteristic values of $\kappa$ are of the order of the inverse beam sizes, i.e.

$$
\kappa \sim \frac{1}{a}
$$

Usually this quantity is much smaller than the typical scale for the variation of the matrix element with respect to the initial momenta. This is the case for $d N_{\text {st }}$ which is determined by the region of large values of $-q^{2}>m^{2}$. Here we can put $\boldsymbol{\kappa}=0$ in $d \sigma(\boldsymbol{\kappa})$ which immediately results in the standard expression for the number of events (25).

However, for the quantity $d N_{\text {nst }}$ determined by the region (6), the variable $q^{2}$ can go through zero. Therefore in this region $q^{2}$ changes considerably if initial momenta are varied on a quantity of the order of $1 / a$. Consequently, transformations (26)-(28) reduce to the following modification:

$$
\frac{1}{\left|q^{2}+i \epsilon\right|^{2}} \rightarrow \frac{1}{t+i \epsilon} \frac{1}{t^{\prime}-i \epsilon}
$$

where

$$
q^{2}=\left(p_{1}-p_{3}\right)^{2}, \quad t=\left(k_{1}-p_{3}\right)^{2}, \quad t^{\prime}=\left(k_{1}^{\prime}-p_{3}\right)^{2} .
$$

Let us expand $t$ and $t^{\prime}$ up to the terms linear in $\boldsymbol{\kappa}$. This gives

$$
t=q^{2}-\lambda\left(q^{2}\right), \quad t^{\prime}=q^{2}+\lambda\left(q^{2}\right)
$$

where

$$
\lambda\left(q^{2}\right)=\boldsymbol{\kappa} \mathbf{Q}, \quad \mathbf{Q}=-\mathbf{p}_{3}+\frac{E_{3}}{E_{1}} \mathbf{p}_{1} .
$$

As the result, the divergent quantity

$$
B=\int_{-m^{2}}^{t_{0}} \frac{d q^{2}}{\left|q^{2}+i \epsilon\right|^{2}}
$$

transforms to

$$
B=\int \frac{d^{3} \kappa d^{3} r}{(2 \pi)^{3}} \mathrm{e}^{i \boldsymbol{} \boldsymbol{r}} \frac{L(\mathbf{r})}{L} \int_{-m^{2}}^{t_{0}} \frac{d q^{2}}{\left[q^{2}-\lambda\left(q^{2}\right)+i \epsilon\right]\left[q^{2}+\lambda\left(q^{2}\right)-i \epsilon\right]}
$$

As has been shown in [2] the main contribution to the integral over $q^{2}$ in eq. (33) is given by the pole

$$
q^{2}=-\lambda\left(q^{2}\right)+i \epsilon \approx-\lambda\left(q^{2}=0\right)+i \epsilon
$$

in the upper half plane. 
In the rest frame of the $\mu^{-}$, the vector $\mathbf{Q}$ is equal to

$$
\mathbf{Q}=-\mathbf{p}_{3}=\mathbf{q}=\omega \mathbf{n}
$$

where $\omega$ is the energy of the real $\left(q^{2}=0\right)$ neutrino and $\mathbf{n}$ is the unit vector which defines direction of motion of the neutrino.

Taking this pole and performing subsequent integrations over $\boldsymbol{\kappa}$ and $\mathbf{r}$, we obtain

$$
B=\frac{\pi}{\omega} \int_{0}^{\infty} \frac{L(r \mathbf{n})}{L} d r
$$

As a result,

$$
d N_{\mathrm{nst}}=d \sigma_{\nu \mu \rightarrow W} \frac{m x}{\omega} d \Gamma \int_{0}^{\infty} L(r \mathbf{n}) d r .
$$

For unpolarized muon beams

$$
d \Gamma=\frac{\Gamma}{\pi}(1-y)(1+2 y) d x d y d \varphi
$$

where $\varphi$ is the azimuthal angle of the vector $\mathbf{q}$.

In the rest frame of the $\mu^{-}$we have

$$
\omega=\frac{m}{2}(1-y), \quad x=\frac{q p_{2}}{p_{1} p_{2}}=\frac{1}{2}(1-y)(1+\cos \theta) .
$$

Here $\pi-\theta$ is the angle between momentum $\mathbf{q}$ of the muonic neutrino and the momentum $\mathbf{p}_{2}$ of the positively charged muon.

Using these equations we express differential width of the $\mu^{-}$as

$$
d \Gamma=\frac{1}{2 \pi \tau}(1-y)^{2}(1+2 y) d y d \cos \theta d \varphi .
$$

Now adding all pieces together and using (see also eq. (21))

$$
d r d \cos \theta d \varphi=\frac{d^{3} r}{r^{2}}
$$

we finally get the following expression

$$
\begin{gathered}
d N_{\mathrm{nst}}=g(y) d y \int v \frac{n_{1}\left(\mathbf{r}_{2}-\mathbf{r}\right)}{4 \pi \tau r^{2}} n_{2}\left(\mathbf{r}_{2}, t\right) d^{3} r d^{3} r_{2} d t \\
g(y)=2(1-y)^{2}(1+2 y) .
\end{gathered}
$$

It is easy to see that after redefinition

$$
y=1-\frac{2 \omega}{m}, \quad \mathbf{r}_{2}-\mathbf{r}=\mathbf{r}_{1}
$$

expression (38) completely coincides with eqs. (16)-(20). 


\section{Remark on the case of a large--size beam}

What happens if conditions (8)-(9) are not satisfied? Though this question is purely academic one for muon colliders, it can have a certain principal interest. There is no general answer in this situation and every such case should be considered separately. In this situation the method of preparation of colliding beams as well as decrease in the numbers of muons and neutrinos during the collision are of great importance. The true consideration in this case would require the modelling of how really unstable particle travels in the collider - it is clear that some characteristics of the collider are likely to enter the final result at this point.

In this section we consider a particular case related to the paper [1]. In this paper the divergent cross section of the process (1) has been regularized by introducing a complex mass for initial unstable particles:

$$
m \rightarrow m-\frac{i}{2} \Gamma
$$

Solving the energy-momentum conservation conditions it is possible to show then that the square of the momentum transfer in the $t$-channel gets small imaginary part:

$$
q^{2} \rightarrow q^{2}-\frac{i}{2} m(1-y) \Gamma
$$

Therefore, the singularity is regularized and the calculation of the cross section starts to be possible. Below we explain the physical meaning of the result obtained in this way in ref. [1] ‥

Let us assume that $\mu^{-}$beam has been prepared much earlier than the collision takes place. This means that almost all muons have managed to decay. In this case every $\mu^{-}$ produces a single $\nu_{\mu}$ with definite distribution over the energy (17) and with the isotropic angular distribution in the rest frame of the $\mu^{-}$. To obtain the results of ref. [1] we also have to assume that the $\mu^{+}$beam is so large that all neutrinos produced in the $\mu^{-}$decay are still inside this beam having a possibility to collide with the muons of the positive charge. This situation is similar to the fast decay of the $\mu^{-}$in a medium of the muons of the positive charge. In such situation the number of events is defined by the cross section $\sigma_{\mu \mu}$ which corresponds to the cross section of the process (3) averaged over the spectrum of neutrinos and their escape angles:

$$
\sigma_{\mu \mu}=\int v w(\omega) d \omega \frac{\sin \theta d \theta d \varphi}{4 \pi} \sigma_{\nu \mu \rightarrow W}\left(s_{\nu \mu}\right)
$$

where $v$ is defined in (20). Using eq. (37) and integrating over $y$ and $\varphi$ we obtain

$$
\sigma_{\mu \mu}=\int_{0}^{1} 4 x(1-x)(2-x) \sigma_{\nu \mu \rightarrow W}(x s) d x .
$$

This result is identical to the one which has been presented in ref. [1].

\footnotetext{
${ }^{5}$ See also ref. [10], where a similar regularization has been used for the process $\pi N^{*} \rightarrow \pi N^{*}$.
} 


\section{Conclusions}

1. Comparing the results of sections 3 and 4, we conclude that the linear beam-size effect obtained in [2] corresponds to the scattering of the $\mu^{+}$beam on the "cloud" of real neutrinos $\nu_{\mu}$ produced in the $\mu^{-} \rightarrow e \bar{\nu}_{e} \nu_{\mu}$ decay. Such interpretation assumes that the colliding beams have finite sizes and that decays of the negative muons on their way to the interaction point produce a $\nu_{\mu}$ cloud which fills the region occupied by $\mu^{+}$beam.

Really amazing is the fact that within perturbative calculations these effects can be found only if one considers a collision of two wave packets which correspond to the beams of the colliding particles. Standard approximations in the scattering theory do not work in this case.

It is interesting to estimate the "formation length" $l_{f}$ of this $\nu_{\mu}$ cloud. In the center of mass frame the angular spread of the muonic neutrinos $\vartheta$ is of the order of $\vartheta \sim 1 / \gamma$. Hence one obtains

$$
l_{f} \sim \frac{a}{\vartheta} \sim \gamma a .
$$

For the discussed parameters of the beams [5] and the total energy $100 \mathrm{GeV}$ one finds $l_{f} \sim 1 \mathrm{~cm}$.

From this we obtain the number of the neutrinos $\nu_{\mu}$ which participate in the collision. It can be estimated as

$$
N_{\mu} \frac{l_{f}}{c \gamma_{\mu} \tau} \sim N_{\mu} \frac{a}{c \tau} \sim 10^{4}
$$

for $N_{\mu} \sim 10^{12}$. This number shows the level of statistical fluctuations which one expects for the $\nu_{\mu} \mu^{+}$collisions.

2. It is interesting to compare the process (1) which has been discussed in this paper and the processes with large impact parameters considered in the review 四, for example, the process

$$
e p \rightarrow e p \gamma
$$

It is not difficult to show that the impact parameters $\varrho$, which give essential contribution to the cross section of the process (43) in the standard calculations, are of the order of

$$
\varrho \lesssim \varrho_{m} \sim \frac{1}{m_{e}} \frac{4 E_{e} E_{p}\left(E_{e}-E_{\gamma}\right)}{m_{e} m_{p} E_{\gamma}} .
$$

Note that the quantity $\varrho_{m}$ can be macroscopically large: for instance, for the collider HERA $\varrho_{m} \gtrsim 1 \mathrm{~cm}$ already for $E_{\gamma} \lesssim 0.3 \mathrm{GeV}$. In this case there is a cloud of equivalent photons (EP) around every proton, these EP scatter on the electrons of the opposite beam. As a result, the interaction of electrons with protons becomes macroscopically nonlocal. In the discussed process (11) similar macroscopically nonlocal interaction between $\mu^{+}$and $\mu^{-}$is due to the cloud of real $\nu_{\mu}$ 's which appears as a result of the muon decay. Certainly, the nature of these clouds is quite different, however, one can describe both processes by means of the same technique.

Distributions of EP and $\nu_{\mu}$ 's in $\varrho$ are absolutely different for both of these processes.

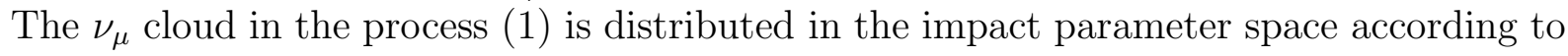
eq. (13), i.e.

$$
d N_{\nu} \propto \frac{d^{2} \varrho}{\varrho} .
$$


This distribution leads (after integration over $\varrho$ in the beam region, $\varrho \lesssim a$ ) to the linear beam-size effect:

$$
d \sigma_{\mu \mu} \propto a .
$$

The distribution of EP has another form

$$
d N_{\mathrm{EP}} \propto \frac{d^{2} \varrho}{\varrho^{2}}
$$

from which the known logarithmic beam-size effect immediately follows:

$$
d \sigma_{e p} \propto \ln a
$$

Let us emphasize that the logarithmic beam-size effect has been already established in experiments [8, 9].

3. Let us finally compare the contribution of real and virtual $\nu_{\mu}$ 's to the total cross section. First, we remind that because of the fixing of the region of the final phase space, the cross section $\sigma_{\text {st }}$ (see eq. (5) and the discussion after it) is completely determined by

virtual $\nu_{\mu}$ 's with $q^{2}<-m^{2}$. Contrary, the contribution of real $\nu_{\mu}$ 's in the intermediate state absolutely dominates in $\sigma_{\text {nst }}$ - the relative contribution of virtual neutrinos to this piece of the cross section can be estimated as $\sim 1 /\left(a \sqrt{t_{0}}\right) \sim 1 /(a m) \sim 10^{-10}$. Let us stress, however, that the approach described in [2] allows to calculate the contribution of virtual neutrinos to $\sigma_{\text {nst }}$ as well as the contribution which appears due to the interference of real and virtual neutrinos.

\section{Acknowledgments}

We are indebted to I. F. Ginzburg, V. S. Fadin, D. Yu. Ivanov, V. V. Serebryakov and V. M. Strakhovenko for useful discussions. K.M. is grateful to the Graduiertenkolleg "Elementarteilchenphysik" of the Mainz University for the support. G.L.K and V.G.S. acknowledge the support of the Russian Fund of Fundamental Research.

\section{References}

[1] I.F. Ginzburg, Preprint DESY 95-168 (1995) and hep-ph 9509314, (unpublished).

[2] K. Melnikov and V.G. Serbo, Phys. Rev. Lett. (in print) and hep-ph 9601221; Preprint MZ-TH-96/03 and hep-ph 9601290.

[3] G.L. Kotkin, S.I. Polityko and V.G. Serbo, Yad. Fiz. 42, 692 (1985).

[4] G.L. Kotkin, V.G. Serbo and A.Schiller, Int. Journ. Modern Physics A7, 4707 (1992).

[5] D. Cline, Nucl. Instrum. Methods 350, 24 (1995); R.B. Palmer, Beam Dynamics Newsletters, 8 (1995).

[6] L. B. Okun, Leptons and Quarks (North-Holland, Amsterdam, 1982).

[7] L.D. Landau and E.M. Lifshitz, Classical Theory of Fields, Pergamon, Oxford, 1962. 
[8] A.I. Blinov et al, Phys.Lett. B113, 423 (1982); V.N. Baier, V.M. Katkov and V.M. Strakhovenko, Yad. Fiz. 36, 163 (1982); A.I. Burov and Ya.S. Derbenev, Preprint 81-64 INP (Novosibirsk, 1981), (unpublished).

[9] K. Piotrzkowski, Zeit. f. Phys. C67, 577 (1995).

[10] R. F. Peierls, Phys. Rev. Lett. 6, 641 (1961). 